\title{
Les leçons de (la) vie de Bertrand Piccard
}

\author{
Jean Martin
}

Notre confrère le psychiatre Bertrand Piccard, né en 1958, est surtout connu, dans le monde, pour ses exploits dans le ciel. Il a beaucoup pratiqué le vol en aile Delta (champion d'Europe de voltige dans ce sport en 1985) et en ULM. En 1992, avec Wim Verstraeten, il a gagné la première course transatlantique en ballon. En mars 1999, toujours en ballon et avec Brian Jones, premier tour du monde, en vingt jours. Depuis 2003, il développe avec d'autres «Solar Impulse», sorte d'immense planeur capable de voler, de jour et de nuit, grâce à l'énergie solaire.

\section{L'expérience lui fait découvrir les apports de situations où, précisément, on n'a pas de contrôle.}

A l'évidence, Bertrand manifeste la dimension de pionnier et d'explorateur de choses nouvelles qu'ont montrée avant lui Auguste, son grand-père, et Jacques, son père. Ce qui est particulier est son intérêt marqué pour la spiritualité. Intérêt (préoccupation, passion?) pour ce que existe au-delà, au-dessus, de ce que nous pouvons voir, sentir, toucher, ou étudier par les techniques scientifiques dures. Dans sa thèse de médecine déjà, il avait interviewé des patients ayant passé par de graves maladies et autres drames, s'intéressant à ce que la souffrance avait pu leur apporter spirituellement ou philosophiquement, en développant chez eux une «pédagogie de l'épreuve».

\section{Le livre}

Une préface de Matthieu Ricard, le moine bouddhiste connu, un avant-propos et douze chapitres. De l'avant-propos: «J'aimerais écrire pour les hommes et les femmes fragilisés par les expériences de la vie, et qui osent écouter ce qui se passe en eux et chez les autres [...] Est-il possible de parler simultanément à ceux qui sont en quête de thérapie, d'exploration, de conseils en management, de spiritualité, afin de laisser la place au questionnement que nous évitons si souvent: comment vivre de façon plus complète notre besoin d'épanouissement - et ceci en se mettant volontairement en état de réceptivité, pour être touchés loin de la protection des a priori et autres convictions.»

\section{Contrôler, ne pas pouvoir le faire, peur de l'inconnu}

Premier chapitre sur les «vents de la vie», sur notre besoin de contrôler, la tendance à se réfugier dans sa zone de confort. «Au lieu d'être conditionnés dans des certitudes, il faudrait saisir qu'il existe des milliers de manières de penser et de réalités différentes. Notre éducation nous présente trop souvent l'existence d'une façon univoque [...] Nous apprenons à nous comporter comme si tout dépendait de nous seuls. Cela fait abstraction de l'imprédictibilité de la vie.» Et de rappeler qu'il ne vaut la peine de chercher à contrôler que ce que nous sommes capables de contrôler. Il parle de sa pratique du vol acrobatique, où le danger auquel on se confronte mène à une perception très aiguë du moment qu'on vit, où il faut être pleinement conscient. «Je me trouvais en porte-à-faux avec la phrase de Descartes Je pense, donc je suis〉. Quand on pense on se disperse, on se projette dans d'autres temps et lieux. Ma seule façon d'exister était de ressentir toutes les impressions de l'instant, la formule devenait pour moi 〈Je ressens, donc je suis`.»

L'expérience lui fait découvrir les apports de situations où, précisément, on n'a pas de contrôle. Première course transatlantique en montgolfière: «Ce fut une révélation. L'absence de puissance et de pouvoir, puisqu'on ne peut compter sur aucun moteur. Permanente gestion de crise.» «Wim Verstaeten et moi nous sommes retrouvés devant un paradoxe. D'un côté, nous étions totalement dépendants du vent qui nous poussait, prisonniers de la météorologie, et de l'autre

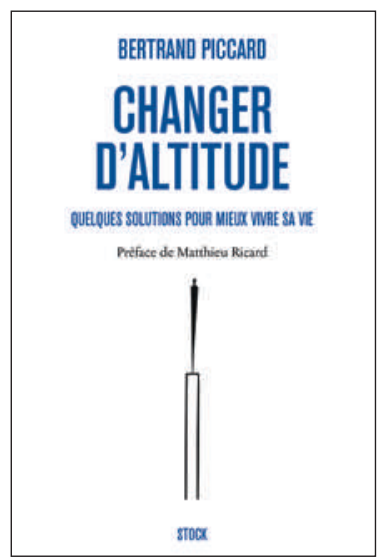

Bertrand Piccard

Changer d'altitude Quelques solutions pour mieux vivre sa vie Paris: Editions Stock; 2014 . 302 pages. 19 EUR. ISBN 978-2-2340-7725-6 


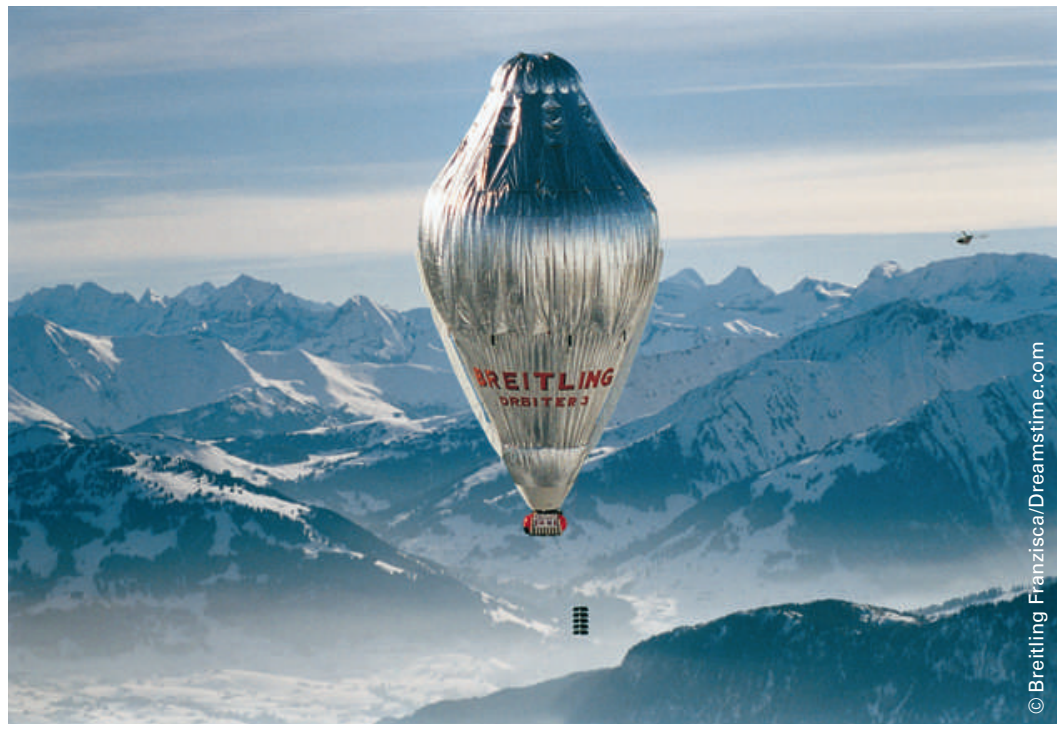

Bertrand Piccard manifeste des dimensions de pionnier et d'explorateur de choses nouvelles: par exemple le tour du monde en ballon en vingt jours.

nous étions totalement libres d'être nous-mêmes, comme affranchis du passé et de l'avenir.» Situations de ruptures.

«Ce que j'ai rapporté de cette aventure est la conviction que notre peur viscérale de l'inconnu est à l'origine d'une majorité de nos souffrances et que notre volonté de contrôle nous fait souvent manquer les cadeaux de l'existence.» «L'incertitude, curieusement, nous permettait une ouverture à l'instant présent, nous sommes devenus plus performants. La relation de confiance entretenue avec le doute et l'inconnu a augmenté notre sentiment de liberté [...]. L'inconnu devenait un allié.»

Chapitre Comment lâcher du lest: importance dans la vie quotidienne de cette mesure de gestion/conduite, la seule disponible dans le vol en ballon. Savoir changer d'altitude (titre de l'ouvrage), de niveau de compréhension ou d'action, vers le haut ou vers le bas.

\section{Un peu de politique - Se vouloir «hérétique»}

Piccard se défend de se mêler de politique, ne croyant plus (comme d'autres) à la pertinence du clivage droite-gauche. Il a toutefois des propos de ce registre: coexistence de ses pulsions de pionnier, naturellement favorable à la libre entreprise, et de souhaits sociaux visant le mieux-être de la société en général. "Je suis conscient que ce que j'écris ici est en contradiction avec la doctrine moderne qui encourage la protection et l'assistance davantage que la prise de risque, le sens des responsabilités. Droits et acquis sont importants, fondamentaux même, mais notre volonté d'en vouloir toujours davantage crée insatisfaction et frustration.»

Nécessité de l'ouverture d'esprit: «Ce qui est passionnant, si nous nous imaginons capables d'évoluer, c'est d'écouter des arguments opposés aux nôtres et d'affaiblir ainsi peu à peu nos propres convictions.»

«La remise en question des convictions communément admises est devenue mon fil rouge. Je suis devenu un hérétique, dans le sens étymologique de celui qui revendique le droit de choisir, par opposition à l'obligation d'accepter les dogmes. Combien de scientifiques, de philosophes, de médecins ont-ils été persécutés pour avoir prôné de lâcher du lest?»

\section{A propos d'éducation}

Donner attention au corps: «L'homme occidental a cru bon, au cours des derniers siècles, de se développer dans sa dimension cérébrale en se distanciant du corps. L'épanouissement spirituel en a souffert; en oubliant notre corps, nous avons perdu la porte d'entrée à la pleine conscience de nous-mêmes, de notre vécu de l'instant présent.»

\section{«La médecine occidentale est un exemple} de captivité dans une unique et même altitude. Elle nous limite encore aujourd'hui à une seule façon de soigner.»

«Les cours de religion devraient ainsi mentionner ce que les autres religions pensent de la nôtre. Le cours d'histoire devrait laisser la parole aux autres cultures. La science devrait présenter notre médecine comme une des multiples façons de soigner, sans occulter les approches chinoise, ayurvédique ou chamanique. [...] On devrait également laisser de la place à l'inconnu, aux sujets pour lesquels personne ne peut apporter d'explications, comme les guérisons spontanées, les miracles, les hypothèses de vie extraterrestre.»

"A ce titre, le domaine des points d'interrogation et des doutes devrait trouver autant de place dans les programmes scolaires que celui des acquis [...] si nous voulons développer chez les jeunes générations les facultés indispensables à trouver leur place dans un monde qui requiert de plus en plus de flexibilité et de moins en moins de certitudes.»

\section{Un substantiel chapitre sur l'hypnose}

Piccard la pratique beaucoup, pour lui (auto-hypnose), avec ses patients et aussi ses co-aéronautes dans leurs longues traversées en ballon. Une pique: avec l'hypnose, le patient est amélioré sans qu'on en connaisse vraiment le mécanisme, mais Piccard la 
préfère à la psychanalyse où le malade ne guérit pas mais comprend pourquoi il souffre... Conseil: «Refouler ses pensées ne crée que des problèmes supplémentaires. Aux autres, il n'est pas adéquat de tout dire, mais à soi-même si. Soyez honnêtes avec vousmême, avouez-vous les plus inavouables pensées qui peuvent vous traverser la tête... Vous n'en serez que plus libres de vous en affranchir.»

Dans la foulée: «La tradition judéo-chrétienne nous apprend que c'est à autrui que nous faisons du bien ou du mal à travers la compassion ou la haine. Or, c'est à nous-mêmes que l'énergie dégagée par nos attitudes fait du bien ou du mal. Quand on pardonne à quelqu'un, c'est soi-même avant tout qu'on libère d'un attachement à une situation négative.»

\section{Spiritualité, ce qui nous dépasse - Vouloir l'écomanité}

Ses trois derniers chapitres traitent des préoccupations affirmées de l'auteur en rapport avec la spiritualité, de la négligence à son égard de la société actuelle, de ses rapports avec la religion. Pour le moins, il vaut la peine d'être confronté à ce que dit quelqu'un comme Bertrand Piccard sur des sujets que beaucoup d'entre nous se gardent d'aborder en société, craignant d'avoir l'air crédules, naïfs voire illuminés. "Quelles que soient notre profession et notre position sociale, la question prioritaire devrait rester celle de nos racines spirituelles et du sens de notre passage sur Terre.» Il plaide pour un concept nouveau d'éco(hu)manité.

\section{A propos de médecine}

«La médecine occidentale est un exemple de captivité dans une unique et même altitude. Elle nous limite encore aujourd'hui à une seule façon de soigner, selon les critères scientifiques statistiquement prouvés. La preuve provient du système même qui a fixé les règles. Je rêve d'une médecine qui ajouterait à sa panoplie de soins scientifiques toutes les approches alternatives, comme autant d'outils différents qui permettraient de soigner nos patients plus efficacement. Et comme rêver ne suffit pas, je l'écris.»

«Les approches médicales issues d'une tradition ésotérique, par opposition à l'allopathie, considèrent que l'être humain est composé d'un corps physique et de plusieurs corps énergétiques de qualités vibratoires différentes. [...] Nous ne sommes pas ici dans la psychosomatique, mais dans l'énergéticosomatique. Les traitements comme l'acupuncture, l'homéopathie, l'ayurvéda, tentent de soigner le niveau subtil en agissant sur la circulation d'énergie.»

Relation soigné-soignant: «Dans notre société, il est trop souvent exigé que le patient s'adapte au système de valeurs de son thérapeute plutôt que l'inverse. Essayez pendant votre prochaine consultation d'expliquer que vous êtes également suivi par un praticien de médecine alternative...»

A propos des expériences et des acquis spirituels des patients ayant traversé des épreuves majeures, il parle de «décalage abyssal entre tous ces témoignages et les préoccupations médicales modernes. Nous sommes en droit de nous demander si les médecins parlent le même langage que leurs patients.» «Les médecins sont-ils formés à laisser une place suffisamment importante aux intérêts philosophiques ou à la vie spirituelle de leurs patients? Nous pouvons répondre par la négative.»

«Le principal obstacle au changement n'est pas le symptôme mais le poids du lest embarqué dans la vie et la peur de l'inconnu. La première action du thérapeute devra stimuler la confiance dans le changement. [...] Lorsque je volais face au vent avec mon aile Delta, je pensais devoir être le pilote de la thérapie. Comme aéronaute, j'ai compris que mon meilleur rôle était celui de météorologue de mon patient. Je n'ai pas à le contrôler mais à lui présenter les différentes manières de penser et d'agir afin qu'il trouve par lui-même la trajectoire qui lui conviendra.»

Well... Bertrand Piccard est un homme et un confrère surprenant. Sans doute ne s'agit-il pas d'abandonner le nécessaire esprit critique parce qu'il a fait des choses hors du commun et vécu des situations que la grande majorité d'entre nous n'approcherons jamais. Cela étant, on ne saurait à mon sens balayer du dos de la main, parce que cela défrise trop, ce qu'il tire d'expériences faites, de convictions tirées d'engagements sérieux dans des voies différentes.

Pour le reste, chacun jugera, qui sait en gardant à l'esprit cette citation encore: «Il me semble que personne, qu'on soit un individu isolé, un chef d'entreprise ou un homme politique, ne peut fonctionner correctement sans être prêt à s'ouvrir au contraire de ce qu'il a toujours pensé. Il n'y a pas de véritable capacité de décision et d'action sans la liberté d'abandonner ses convictions, de raisonner en dehors de ce que nous avons appris et qui nous a conditionnés.» 